\title{
Automated Collection of Electronic Health Record Healthy Weight Data for Surveillance
}

\author{
Sean P. Mikles ${ }^{1}$, Jennifer L. Foltz ${ }^{2}$, Ian Painter ${ }^{1}$ and William B. Lober*1 \\ 'University of Washington Schools of Medicine, Nursing and Public Health, Seattle, WA, USA; ${ }^{2}$ Centers for Disease Control and \\ Prevention, Atlanta, GA, USA
}

\section{Objective}

To demonstrate the feasibility of using healthy weight (HW) IT standards in public health surveillance through the collection and visualization of patient height, weight and behavioral data.

\section{Introduction}

Clinical data captured in electronic health records (EHR) for patient health care could be used for chronic disease surveillance, helping to inform and prioritize interventions at a state or community level. While there has been significant progress in the collection of clinical information such as immunizations for public health purposes, greater attention could be paid to the collection of data on chronic illness. Obesity is a chronic disease that affects over a third of the US adult population ${ }^{1}$, making it an important public health concern. Both HL7 v.2.5.1 ${ }^{2}$ and Clinical Document Architecture (CDA) messages ${ }^{3}$ can be used to facilitate the collection of HW EHR data. These standards include anthropometric and demographic information along with the option to transmit behavioral, continuity of care, community resource identification and care plan information. We worked with vendors participating in the Integrating the Healthcare Enterprise initiative (IHE) in developing, testing and showcasing scenarios to facilitate system development, increase the visibility of HW standards and demonstrate potential usages of obesity-related information.

\section{Methods}

The IHE initiative showcases integration of health information systems within and across institutions. Integration profiles, which describe the use of standards to implement data transactions that satisfy specific use cases, are developed by committees, implemented and tested in a multi-vendor Connectathon, and then demonstrated at national and international meetings. This process was followed to illustrate the feasibility and potential uses of HW data. A system was developed to receive and manage data from v.2.5.1 and CDA HW messages sent by clinical systems. Potential uses of HW data were conceptualized through project team collaborations and discussions with clinical and public health stakeholders and used to create a HW dashboard. These uses included stratified displays of overall weight metrics by demographics and locations, displays of the distribution of behavioral risk factors such as diet and physical activity, and the display of the metrics of a hypothetical provider's cohort of patients as compared to the entire community.

\section{Results}

The HW surveillance data collection and dashboard visualizations have been demonstrated at the 2014 Health Information and Management Systems Society (HIMSS, Orlando FL) and Public Health Informatics (PHIC, Atlanta GA) conferences. The visualizations were presented as part of an integrated walkthrough with vendor partners, which included the entering of clinical information in an EHR, the transmission of the information to the HW Information System, and the display of graphs and visualizations of a hypothetical data set based on metrics from the state of Washington. The dashboard and displays for HIMSS and PHIC may be viewed at http://ihe2014.cirg. washington.edu (accessed Sept 7, 2014).

\section{Conclusions}

This project demonstrated HW IT standards based collection of weight-related data from EHRs and explored data visualizations derived from those data. HW surveillance could identify the need for, shape the design of, and support monitoring and evaluation of public health interventions. Future work can expand these demonstrations into limited pilot projects to investigate the efficacy of EHR-based HW data collection.

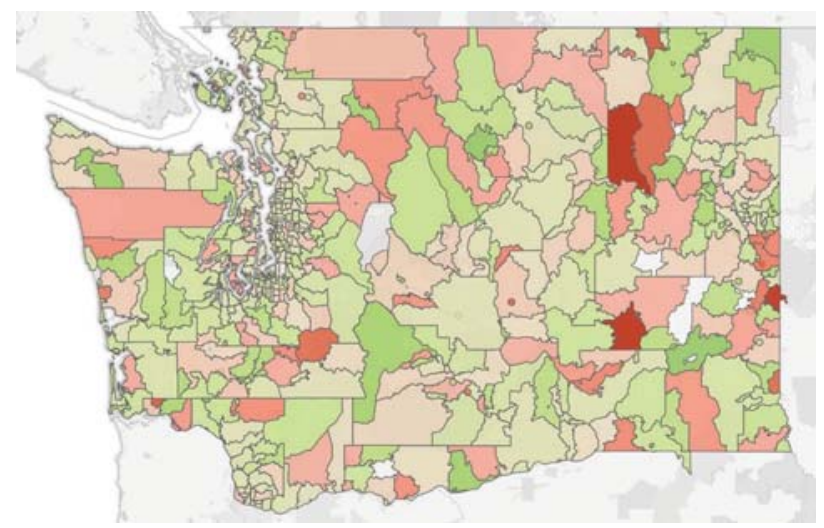

Visualizations, like this map of simulated BMI data by zip code, can help public health agencies target interventions.

\section{Keywords}

Healthy Weight; Obesity; Chronic Disease; Informatics; Surveillance

\section{Acknowledgments}

This work was supported by funding from CDC/National Center for Chronic Disease Prevention and Health Promotion and in part by NIH NLM Training Grant Nr. T15LM007442.

\section{References}

1. CDC. (2011). Obesity: Halting the Epidemic by Making Health Easier At A Glance 2011. [Online]. Available: http://www.cdc.gov/ chronicdisease/resources/publications/AAG/obesity.htm. [6 Sep 2014].

2. HL7. HL7 Version 2.5.1 Implementation Guide: Height and Weight Report, Release 1. [Online]. Available: http://www.hl7.org/implement/ standards/product_brief.cfm?product_id=315. [6 Sep 2014]

3. IHE. IHE Quality, Research and Public Health Technical Framework Supplement for Healthy Weight. [Online]. Available: http://www.ihe. net/uploadedFiles/Documents/QRPH/IHE_QRPH_Suppl_HW.pdf. [6 Sep 2014]

\footnotetext{
*William B. Lober

E-mail: lober@uw.edu
} 